\title{
Forced large amplitude periodic vibrations of nonlinear Mathieu resonators for microgyroscope applications
}

\author{
N. Kacem ${ }^{* a, b}$, S. Hentz ${ }^{\text {b }}$ S. Baguet ${ }^{\mathrm{a}}$, R. Dufour ${ }^{\mathrm{a}}$ \\ ${ }^{a}$ Université de Lyon, CNRS \\ INSA-Lyon, LaMCoS UMR5259, F-69621, Villeurbanne, France \\ ${ }^{b}$ CEA/LETI - MINATEC, Grenoble, France
}

\begin{abstract}
This paper describes a comprehensive nonlinear multiphysics model based on the Euler-Bernoulli beam equation that remains valid up to large displacements in the case of electrostatically actuated Mathieu resonators. This purely analytical model takes into account the fringing field effects and is used to track the periodic motions of the sensing parts in resonant microgyroscopes. Several parametric analyses are presented in order to investigate the effect of the proof mass frequency on the bifurcation topology. The model shows that the optimal sensitivity is reached for resonant microgyroscopes designed with sensing frequency four times faster than the actuation one.
\end{abstract}

Key words:

Microgyroscope, periodic vibrations, nonlinear dynamics, Mathieu resonator, bifurcation topology, scale factor

\section{Introduction}

Microelectromechanical gyroscopes have attracted lots of attention due to their small size, batch fabrication, Integrated Circuit (IC) compatibility, low cost, and acceptable moderate performance for most applications. They can be used either as a low-cost miniature companion with micromachined accelerometers to provide heading information for inertial navigation purposes or in other areas, including automotive applications for ride stabilization and rollover detection; some consumer electronic applications, such as video-camera stabilization, virtual reality, and inertial mouse for computers; robotics applications; and a wide range of military applications [1,2].

Micromachined gyroscopes typically rely on the coupling of an excited vibration mode into a secondary mode due to the Coriolis acceleration based on the combination of vibration of a proofmass and an orthogonal angular-rate input. As shown in Fig. 1, when the gyroscope is subjected to an angular rotation, a sinusoidal Coriolis force is induced in the direction orthogonal to the drive-mode oscillation at the driving frequency.

${ }^{*}$ Corresponding author. Tel.: +3343878 01 27; fax: +33438782434.

Email address: najib. kacem@insa-lyon.fr (N. Kacem) 


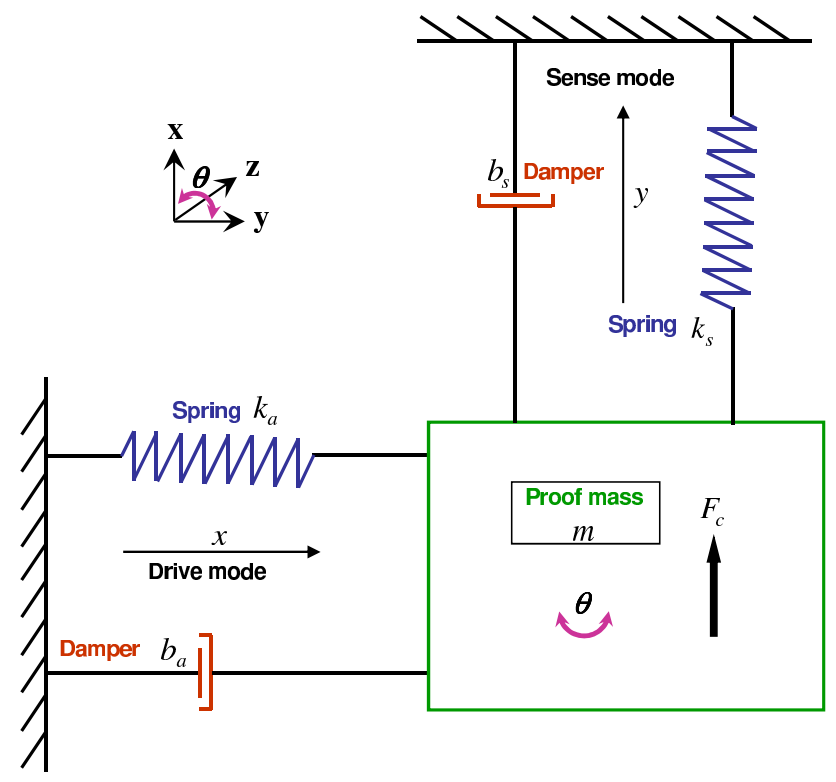

Figure 1: Lumped parameter model of a vibratory gyroscope.

To achieve high sensitivity in conventional micro rate gyroscopes based on harmonic oscillators, the drive and the sense resonant frequencies are typically designed and tuned to match, and the device is controlled to operate at or near the peak of the response curve (where amplitude is defined by the Q-factor) [3]. However, current micro fabrication processes produce asymmetries causing frequency mismatching between modes, translating to drastic loss of sensitivity [4]. Although solutions to overcome frequency mismatching have been pursued [5, 6], many of them involve adding complexity to the system by including additional controllers, additional degrees of freedom [7] or utilizing multiple drive mode oscillators [8].

Due to the complexity of the proposed control schemes $[5,6]$, alternate approaches were considered. One of them is the use of resonant sensing [9] of the Coriolis force instead of displacement sensing employed in most conventional microgyroscopes. Profiting from the high sensitivity of the resonant detection, the matching of the drive and the sense frequencies is not mandatory to achieve a high resolution. Consequently, the number of states that have to be simultaneously controlled and the number of variables that require identification are much smaller and the dynamics is simplified from a minimally two-dimensional system to a series of coupled one-dimensional mass-spring-damper systems. Nevertheless, resonant sensing suffers from the soon occurrence of nonlinearities [10-13] which reduces the gyroscope performances. Moreover and unlike resonant accelerometers, the sensing parts are Mathieu resonators since their stiffnesses are modulated periodically by a time-varying Coriolis force at the proof mass frequency.

In this paper, a typical resonant gyroscope is described and equations of motion are derived for both actuation and sensing elements. Then, the nonlinear dynamics of electrostatically driven Mathieu resonators is investigated for resonant gyroscope applications using a complete model which includes the mechanical nonlinearities, nonlinear electrostatic terms up to the fifth order as 
well as the Coriolis parametric excitation. The model is based on the Galerkin expansion method coupled with a perturbation technique; the resonant and periodic case under primary excitation has been considered. The main idea is to provide practical rules for microgyroscopes designers based on the proof mass frequency effect analysis in order to enhance the scale factor of the resonant sensor.

\section{The Resonant Gyroscope}

The resonant gyroscope, as its name implies, utilizes resonant sensing as a basis for Coriolis force detection. In its simplest form, the device consists of three resonating elements, a proof mass vibrating in the tens of kiloHertz and two resonating sense elements, with a designed resonant frequency, generally, an order of magnitude higher than that of the proof mass in order to avoid possible parametric instabilities in detection. Although the principle is general enough to accommodate devices that sense rotation rate about in-plane axes with minor modifications (such as driving the proof mass perpendicular to the substrate), the focus of this paper concerns gyroscopes that sense rotation rate about a single axis orthogonal to the plane of the device substrate. In addition, gyroscope topologies for a dual-mass to cancel common-mode acceleration signals and gyroscope suspensions to reduce quadrature error carry over for a resonant gyroscope. A schematic of the z-axis resonant gyroscope is shown in Fig. 2.

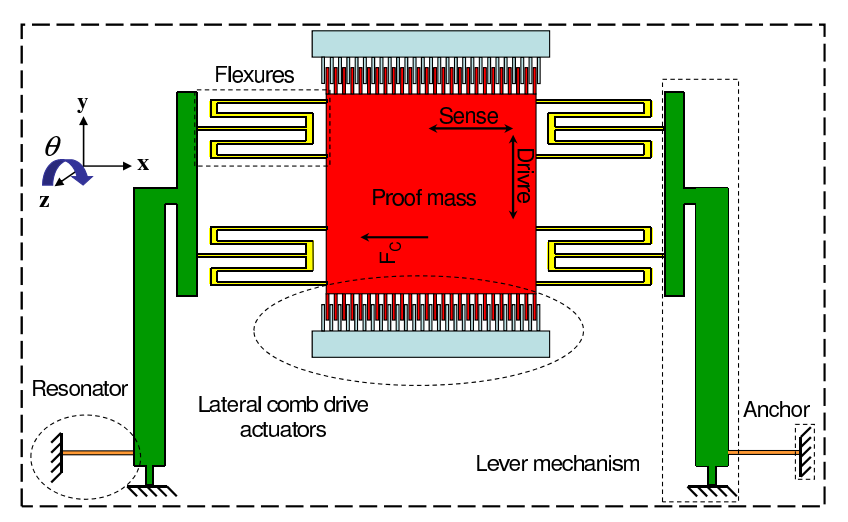

Figure 2: Schema of a simple mass resonant gyroscope.

The device consists of a proof mass suspended by flexures attached to lever mechanisms [14] for Coriolis force amplification. The proof mass is driven along the $y$ axis using embedded lateral comb drive actuators. If an external rotation is applied to the chip about the z-axis, the Coriolis forces acting on the proof mass is transmitted to the lever mechanisms that amplify these forces prior to its being communicated axially onto two resonators placed on each side of the proof mass for a differential output. The two resonators vibrate out of phase and parallel to the direction of motion of the proof mass. The periodic compression and tension of the resonators by the Coriolis force at the proof mass drive frequency modulates the resonant frequency of these force sensors. Each force sensor comprises mechanical resonator shown in Fig. 3, electrostatically actuated and 
embedded in the feedback loop of an oscillator circuit. Thus, by demodulating the oscillation frequency, the rotation rate applied to the device can be estimated.

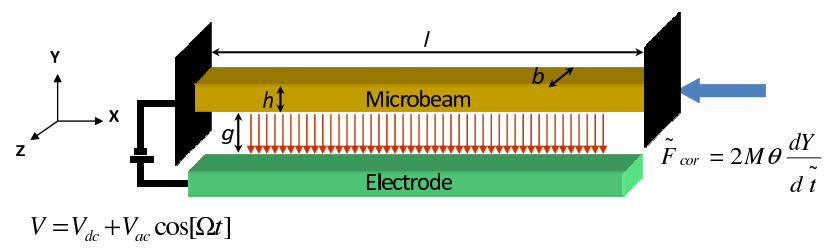

Figure 3: Sketch of a Mathieu resonator.

The dynamics of the device (Figs. 2 and 3) can be described by a series of coupled differential equations. The proof mass dynamics can be described for most part by a classical spring-massdamper equation. The dynamics of the resonator subjected to an axial time-varying Coriolis force is described by a nonlinear Mathieu partial differential equation. The respective equations can be written:

$$
\begin{array}{r}
\frac{d^{2} Y(\tilde{t})}{d \tilde{t}^{2}}+\frac{\tilde{\delta}}{Q} \frac{d Y(\tilde{t})}{d \tilde{t}}+\tilde{\delta}^{2} Y(\tilde{t})=\frac{F_{e}}{M} \cos \tilde{\delta} \tilde{t} \\
E I \frac{\partial^{4} \tilde{w}(\tilde{x}, \tilde{t})}{\partial \tilde{x}^{4}}+\rho b h \frac{\partial^{2} \tilde{w}(\tilde{x}, \tilde{t})}{\partial \tilde{t}^{2}}+\tilde{c} \frac{\partial \tilde{w}(\tilde{x}, \tilde{t})}{\partial \tilde{t}} \\
-\frac{E b h}{2 l} \int_{0}^{l}\left[\frac{\partial \tilde{w}(\tilde{x}, \tilde{t})}{\partial \tilde{x}}\right]^{2} d \tilde{x} \frac{\partial^{2} \tilde{w}(\tilde{x}, \tilde{t})}{\partial \tilde{x}^{2}} \\
-\left[\tilde{N}+A_{l} \tilde{F}_{c} \cos \tilde{\delta} \tilde{t}\right] \frac{\partial^{2} \tilde{w}(\tilde{x}, \tilde{t})}{\partial \tilde{x}^{2}}= \\
\frac{1}{2} \varepsilon_{0} \frac{b C_{n}[V d c+V a c \cos (\tilde{\Omega} \tilde{t})]^{2}}{(g-\tilde{w}(\tilde{x}, \tilde{t}))^{2}} \\
\tilde{F}_{c}=2 M \theta \frac{d Y(\tilde{t})}{d \tilde{t}}
\end{array}
$$

where $\tilde{x}$ is the position along the resonator length, $E$ and $I$ are the Young's modulus and moment of inertia of the cross section. $\tilde{N}$ is the applied tensile axial force due to the residual stress on the silicon, $\tilde{t}$ is time, $\rho$ is the material density, $h$ is the microbeam thickness, $g$ is the capacitor gap width, and $\varepsilon_{0}$ is the dielectric constant of the gap medium. The last term on the left-hand side of Eq. (2) represents an approximation of the electric force assuming a complete overlap of the area of the microbeam and the stationary electrode including the edge effects by the coefficient $C_{n}$ [15].

Furthermore, $\tilde{Y}$ is the mass displacement along the vertical axis, $M$ is the mass of the drive part of the resonant gyroscope, $\delta$ is its resonance frequency, $Q$ the drive quality factor, $F_{e}$ is the electrostatic force induced by the lateral comb drive actuators, $A_{l}$ is the amplification coefficient of the Coriolis $F_{c}$ force due to the lever mechanism. The perturbation term $A_{l} \tilde{F}_{c} \cos \tilde{\delta} \tilde{t}$, which represents a modulation of the spring constant of the resonant sensor at the gyroscope drive frequency $\left(\frac{\tilde{\delta}}{2 \pi}\right)$, is directly contributed by the amplified Coriolis force impinging axially on the resonator. Thus, the Coriolis force $\left(\tilde{F}_{c}\right)$ modulates the spring constant of the resonator system. 
Clearly, Eq. (2) governing the micro-beam resonator dynamics is similar to the equation of motion of the sensing parts in the case of a resonant accelerometer [10]. The only difference is the time-varying term proportional to the Coriolis force that modulates the spring constant of the resonator. Obviously, for a very low actuation frequency $\tilde{\delta}$ with respect to the resonator natural frequency, the Coriolis force is quasi-static and then sensing dynamics for both resonant inertial sensors become equivalent.

Note that the two resonators placed on each side of the structure experience an equal and opposite axial force. The output of the device is the resonant frequency shift difference $(\Delta f=$ $\Delta f_{1}-\Delta f_{2}$ ) between the two resonators, measured at the gyroscope proof mass drive frequency.

\subsection{Actuation part}

The details about the dimensions (proof mass+spring) are described in Fig. 4.
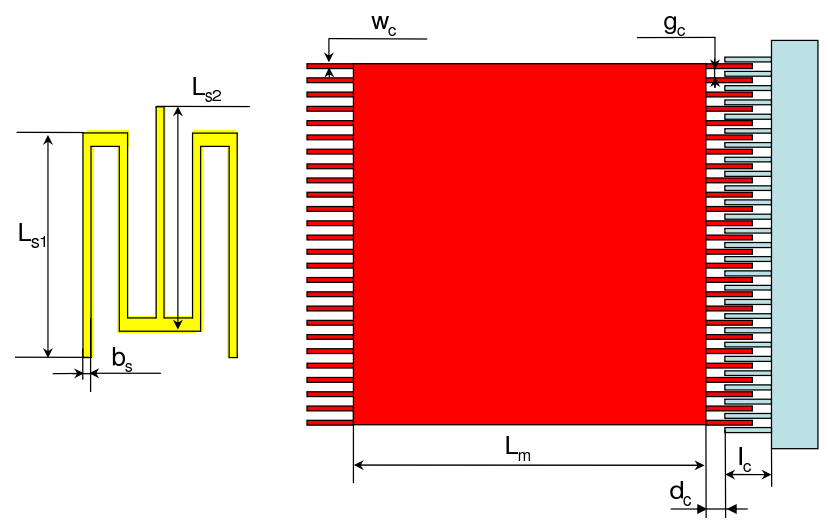

Figure 4: Proof mass and spring designs for a resonant gyroscope.

An equivalent mechanical model was used in order to compute analytically the stiffness $K_{m}$ of the spring-mass-damper system as follow:

$$
K_{m}=\frac{8 E e_{M} b_{s}}{3 L_{s 1}+L_{s 2}}
$$

where $e_{M}$ is the MEMS level thickness (the resonator thickness could be smaller than the proof mass, springs and lever mechanisms thickness which is the case of a M\&NEMS gyroscope). The natural frequency of the actuation system is then:

$$
\tilde{\delta}=\sqrt{\frac{8 E b_{s}}{\rho L_{m}^{2}\left(3 L_{s 1}^{3}+L_{s 2}^{3}\right)}}
$$

The electrostatic force generated by the comb drive actuators is:

$$
\begin{array}{r}
F_{e}=\frac{\partial U}{\partial g_{c}}=n \frac{\epsilon_{0} e_{M}\left(V m_{d c}+V m_{a c} \cos \tilde{\delta} \tilde{t}\right)^{2}}{g_{c}} \\
n=\left\lfloor\frac{2 L_{m}+g_{c}-W_{c}}{g_{c}+W_{c}}\right\rfloor
\end{array}
$$


where \lfloor\rfloor denotes the floor function, $n$ is the number of interdigitated fingers, $U$ is the energy associated with the applied electric potential (a drive voltage $V m_{a c}$ and a polarization $V m_{d c}$ ), $W_{c}$ is the width of a finger, $g_{c}$ is the gap between two fingers as shown in Fig. 4.

The quality factor of the mass-spring-damper system being very high $\left(10^{4}<Q_{m}<10^{6}\right)$, the static displacement is negligible with respect to the dynamic displacement and then the proof mass displacement at resonance can be formulated as follows:

$$
Y_{M}=\frac{Q_{m} F_{e}}{\rho \tilde{\delta}^{2} L_{m}^{2} e_{M}}
$$

As the proof mass is actuated using a lateral comb drive topology and is allowed to displace along the sense direction as well, then it becomes susceptible to a pull-in like phenomenon since the actuator topology looks more like a parallel-plate capacitor along the sense direction. As a result, motion along the sense direction due to non-idealities or asymmetries in the electromechanical structure places an upper limit on the actuation voltage and the displacement that can be allowed along the sense axis. As it depends on the resonator stiffness along the $X$ axis, this issue is negligible for our designs of resonant gyroscope. If an external rotation rate $\theta\left({ }^{\circ} / s\right)$ is applied to the chip about the z-axis, the Coriolis force acting on the proof mass is:

$$
\tilde{F}_{c}=\frac{2 \pi}{180} L_{m}^{2} e_{M} \rho \theta \tilde{\delta} Y_{M}
$$

\subsection{Lever mechanism}

Since a high scale factor is required for a low noise sensor, a lever mechanism suitable for surface micromachined technology is used in order to amplify the Coriolis force acting on the proof mass. It is made of flexural pivots for leverage and to link to the input $\left(F_{c}\right)$ and output $\left(F_{c a}\right)$ forces (true pivots are unavailable in the fabrication processes). As long as the torsional stiffness of the flexures is not too high, the structure will effectively approximate a lever, magnifying the input force communicated to the resonators and increasing the scale factor of the sensor.

The model for determining the actual amount of magnification provided by the lever is shown in Fig. 5. The behavior of the system must be solved by simultaneously solving for the vertical deflection and rotation of the system which gives the following equations:

$$
\begin{array}{r}
X=\frac{1}{k_{p i v}}\left(1-\frac{k_{r} L_{a 1} L_{a 2}}{k_{\Phi}+k_{r} L_{a 2}^{2}}\right) \tilde{F}_{c} \\
\Phi=\frac{L_{a 1}}{k_{\Phi}+k_{r} L_{a 2}^{2}} \tilde{F}_{c} \\
\tilde{F_{c a}}=\frac{k_{r} L_{a 1} L_{a 2}}{k_{\Phi}+k_{r} L_{a 2}^{2}} \tilde{F}_{c}
\end{array}
$$

where $X$ is the deflection of the structure, $\Phi$ is the rotation of the structure, $\tilde{F_{c a}}$ is the output force (the amplified Coriolis force), $k_{p i v}$ is the vertical stiffness of the pivot beam, $k_{r}$ is the stiffness of the output structure (the resonator), $k_{\Phi}$ is the sum of the link rotational stiffnesses $k_{r, \Phi}$ and $k_{p i v, \Phi}$, $L_{a 2}$ is the distance from the pivot to the output, $L_{a 1}$ is the distance from the pivot to the input and $\tilde{F}_{c}$ is the input force (the Coriolis force acting on the proof mass). 


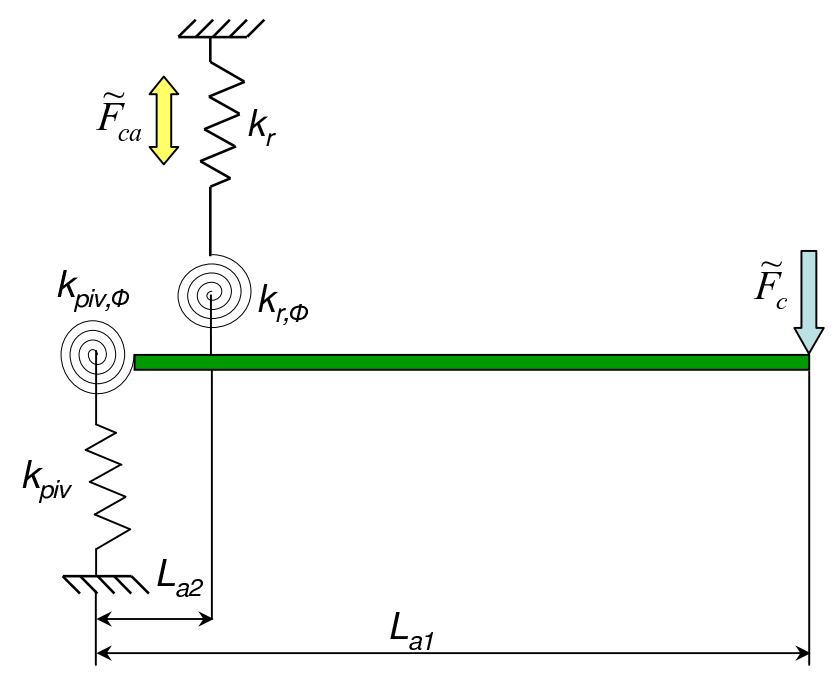

Figure 5: Model used to predict leverage force magnification.

In the ideal case, the torsional stiffness of the flexures is zero and the vertical stiffness of the pivot is infinite, and above equations to:

$$
\begin{array}{r}
X=0 \\
\Phi=\frac{L_{a 1}}{k_{r} L_{a 2}^{2}} \tilde{F_{c}} \\
\tilde{F_{c a}}=\frac{L_{a 1}}{L_{a 2}} \tilde{F}_{c}
\end{array}
$$

In order to obtain the maximum possible amplification, the position of the resonator with respect to the lever mechanism structure has been optimized by FE simulations.

\section{Model}

A sketch of a Mathieu resonator is presented in Fig. 3. In order to model and investigate the nonlinear dynamics of such electromechanical structure, the nonlinear partial differential equation (2) is considered with the following boundary conditions:

$$
\tilde{w}(0, \tilde{t})=\tilde{w}(l, \tilde{t})=\frac{\partial \tilde{w}}{\partial \tilde{x}}(0, \tilde{t})=\frac{\partial \tilde{w}}{\partial \tilde{x}}(l, \tilde{t})=0
$$

The Mathieu equation has been widely studied in the context of parametric resonance. Newman et al [16] investigated the dynamics of a parametrically excited partial differential equation and particularly the dependence of the steady state behavior on parameter values and initial conditions. In [17], a new technique derived from [18] based on an approximate realization of the method of averaging has been used to tackle weakly nonlinear Mathieu equations whose unperturbed dynamics is close to points corresponding to simple resonances between response and parametric forcing. 
Rand et al [19] constructed analytical expressions for the transition curves of the quasiperiodic Mathieu equation in the vicinity of the resonance $2: 2: 1$ using a double-perturbation procedure. In [20], the interaction of subharmonic resonances in the nonlinear quasiperiodic Mathieu equation has been investigated. Belhaq and fahsi [21] showed that in the vicinity of the $2: 1$ and $1: 1$ resonances in a fast harmonically excited Van Der Pol-Mathieu-Duffing oscillator, fast harmonic excitation can change the nonlinear characteristic spring behavior from softening to hardening and causes the entrainment regions to shift. In [22], the motion of a sample automotive belt-pulley system subjected to tension fluctuations governed by a Mathieu-Duffing equation was theoretically and experimentally investigated.

Note that the transition curves of a linear Mathieu equation (Fig. 6) can be approximately plotted using a perturbation technique. The number of tongues of instability corresponds to the truncation order of the asymptotic expansion. Figure 6 displays three instability tongues that correspond to a third order expansion of the perturbation technique. The curves are determined analytically using the Floquet theory for small Coriolis forces. Here, $\Delta$ is the ratio between the proof mass frequency and the resonator frequency and $F_{c a}$ is the dimensionless input time-varying force axially applied to the resonator. These instability tongues emanate from the points $\frac{1}{\Delta}=\frac{n}{2}$ on the $\frac{1}{\Delta}$ axis. Dufour and Berlioz [23] showed that the dynamic stability of parametrically exited beams depends on the type of parametric excitation, the forcing frequencies and the boundary conditions and demonstrated that the existence of the instability zone is in relationship with the topology of the modal geometric stiffness matrices due to axial force and torque.

Figure 6 shows the instability chart which correspond to the Strutt diagram for $\frac{1}{\Delta}$. Inside the tongues, the resonator displacement grows exponentially in time. Outside the tongue, the displacement becomes the sum of terms each of which is the product of two periodic (sinusoidal) functions with generally incommensurate frequencies, that is, the displacement is a quasiperiodic function of time. Also, the resonator is very weakly damped (high quality factors) which makes

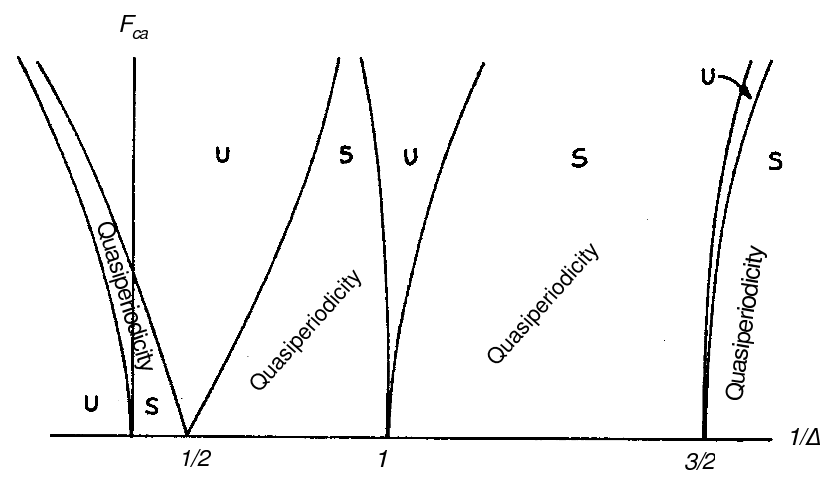

Figure 6: Transition curves in a linear autonomous Mathieu equation. $S$ denotes stable quasiperiodic domains and $U$ denotes the unstable domains.

these transition curves approximately valid for a damped linear Mathieu equation. Figure 6 shows unbounded solutions to Mathieu's equation which can result from resonances between the forcing frequency and the oscillator's unforced natural frequency. However, real physical systems do not exhibit unbounded behavior. The difference lies in the fact that the Mathieu equation is linear. The 
effects of nonlinearity can be explained as follows: as the resonance causes the amplitude of the motion to increase, the relation between period and amplitude (which is a characteristic effect of nonlinearity) causes the resonance to detune, decreasing its tendency to produce large motions.

The equation that governs the Mathieu resonator is a nonlinear partial differential equation under parametric and external excitation and consequently, further complicated than those already studied in literature. In addition, the structure of the stability regions of the quasiperiodic Mathieu equation is much more complicated than for the Mathieu equation.

Belhaq et al. [24] and Guennoun et al. [25] consider a homogeneous Mathieu equation with quasiperiodic linear coefficients and a constant nonlinear coefficient. The small parameter technique of multiple scales is applied twice to the system to obtain an approximate time-invariant system. In another study (see Belhaq and Houssni, [26] the system under investigation contains quadratic and cubic nonlinearities as well as parametric (linear terms) and external excitations of incommensurate frequencies. The small parameter techniques of generalized averaging and multiple-scale perturbation are employed to obtain a solution. Rand and his associates [20, 27, 28] analyze a linear homogeneous quasiperiodic Mathieu equation via several methods such as numerical integration, Lyapunov exponents, regular perturbation, Lie transform perturbation and harmonic balance.

The purpose of this paper is to provide practical rules for MEMS designers in order to enhance the performances of resonant microgyroscopes. Since quasiperiodicity is an undesirable phenomenon which can lead to chaotic oscillations, we restrict our investigations to the periodic motions of the forced nonlinear Mathieu equation.

Following Rand and Morrison [27], in our case for an external excitation tuned around the resonator primary resonance and by analogy, the Mathieu equation is quasiperiodic when $\Delta \neq \frac{2-m}{n}$ for $\Delta \in[0,1], m \in \mathbb{Z}$ and $n \in \mathbb{N}$. Consequently, the quasiperiodic domains in the $\left(\Delta, F_{c a}\right)$ plan are very limited for low frequencies actuation $(\tilde{\delta})$ with respect to the periodic motions. This ensures the generality of our parametric analysis of the gyroscope sensitivity out of quasiperiodicity. However, a first order averaging method is valid only for low Coriolis forces which ensure a negligible effect of the superharmonic resonances at each $\Delta<1$. Otherwise, high order averaging is required. For simplicity, all these conditions are assumed to be satisfied.

\subsection{Nondimensionalization}

For convenience and equations simplicity, the following nondimensional variables are introduced:

$$
w=\frac{\tilde{w}}{g}, x=\frac{\tilde{x}}{l}, t=\frac{\tilde{t}}{\tau}
$$


where $\tau=\frac{2 l^{2}}{h} \sqrt{\frac{3 \rho}{E}}$. Substituting Eq. (17) into Eqs. (2) and (16), yields:

$$
\begin{array}{r}
\frac{\partial^{4} w}{\partial x^{4}}+\frac{\partial^{2} w}{\partial t^{2}}+c \frac{\partial w}{\partial t}-\alpha_{2} \frac{[V d c+V a c \cos (\Omega t)]^{2}}{(1-w)^{2}}= \\
{\left[N+F_{c} \cos \delta t+\alpha_{1} \int_{0}^{1}\left[\frac{\partial w}{\partial x}\right]^{2} d x\right] \frac{\partial^{2} w}{\partial x^{2}}} \\
w(0, t)=w(1, t)=\frac{\partial w}{\partial x}(0, t)=\frac{\partial w}{\partial x}(1, t)=0
\end{array}
$$

The parameters appearing in Eq. (18) are:

$$
\begin{gathered}
c=\frac{\tilde{c} l^{4}}{E I \tau}, \quad N=\frac{\tilde{N} l^{2}}{E I}, \quad F_{c}=A_{l} \frac{\tilde{F}_{c} l^{2}}{E I}, \quad \delta=\tilde{\delta} \tau \\
\alpha_{1}=6\left[\frac{g}{h}\right]^{2}, \quad \alpha_{2}=6 C_{n} \frac{\varepsilon_{0} l^{4}}{E h^{3} g^{3}}, \quad \Omega=\tilde{\Omega} \tau
\end{gathered}
$$

\subsection{Reduced order model}

In practice, the quality factors $Q$ of Mathieu resonators are in the range of $10^{3}-5.10^{4}$ which makes the static displacement negligible with respect to the dynamic displacement of the microbeam. A reduced-order model is generated by modal decomposition transforming Eq. (2) into a finite-degree-of-freedom system consisting of nonlinear Mathieu ordinary differential equations in time. The undamped linear mode shapes of the straight microbeam are used as basis functions in the Galerkin procedure. To this end, the deflection is expressed as:

$$
w(x, t)=\sum_{k=1}^{n} a_{k}(t) \phi_{k}(x)
$$

where $a_{k}(t)$ is the $k^{\text {th }}$ generalized coordinate and $\phi_{k}(x)$ is the $k^{\text {th }}$ linear undamped mode shape of the straight microbeam, normalized such that $\int_{0}^{1} \phi_{k} \phi_{j}=\delta_{k j}$ where $\delta_{k j}$ is the Kronecker symbol. The linear undamped mode shapes $\phi_{k}(x)$ are governed by:

$$
\begin{array}{r}
\frac{d^{4} \phi_{k}(x)}{d x^{4}}=\lambda_{k}^{2} \phi_{k}(x) \\
\phi_{k}(0)=\phi_{k}^{\prime}(0)=\phi_{k}^{\prime}(1)=\phi_{k}^{\prime \prime}(1)
\end{array}
$$

Here, $\lambda_{k}$ is the $k^{\text {th }}$ natural frequency of the resonator. Since some high order nonlinearities proves to be relevant by enabling the capture of 5 possible amplitudes for a given frequency in the mixed behavior [29], the electrostatic force in Eq. (18) is expanded in a fifth order Taylor series. Then, Eq. (21) is substituted into the resulting equation, Eq. (22) is used to eliminate $\frac{d^{4} \phi_{k}(x)}{d x^{4}}$, and the outcome is integrated from $x=0$ to 1 . Thus, a system of coupled nonlinear Mathieu ordinary 
differential equations in time is obtained. The $D C$ voltage, which is generally at least ten times higher than the $A C$ voltage, makes the second harmonic negligible. Also, the first mode should be the dominant mode of the system. According to this assumption, the study can be restricted to the case $n=1$. then, it gives:

$$
\begin{array}{r}
\ddot{a}_{1}+c \dot{a}_{1}+\left(500.564-2 \alpha_{2} \xi_{0}+12.3 N\right) a_{1} \\
+\alpha_{2} \xi_{0}\left(0.831+4 a_{1}{ }^{2}+13.255 a_{1}{ }^{4}\right) \\
+12.3 F_{c} a_{1} \cos \delta t-23.17 \alpha_{2} \xi_{0} a_{1}{ }^{5} \\
+\left(151.354 \alpha_{1}-7.403 \alpha_{2} \xi_{0}\right) a_{1}^{3}=0
\end{array}
$$

where $\xi_{0}=V_{d c}^{2}+V_{a c} \cos \Omega t$. To analyse the equation of motion (24), it proves convenient to invoke perturbation techniques [30] which work well with the assumptions of "small" excitation and damping, typically valid in MEMS resonators. Nevertheless, in order to avoid quasiperiodicity, we chose $\frac{\delta}{\omega_{n}} \in \mathbb{Q} \cap[0,1]$ and we assume that the Coriolis forces are weak enough to make the possible superharmonic resonances negligible with respect to the fundamental primary resonance.

Since near-resonant behavior is the principal operating regime of the proposed system, a detuning parameter, $\sigma$ is introduced, as given by:

$$
\Omega=\omega_{n}+\varepsilon \sigma
$$

where $\omega_{n}=\sqrt{500.564+12.3 N-2 V_{d c}^{2} \alpha_{2}}$. Separating the resulting equations and averaging them over the period $\frac{2 \pi}{\Omega}$ in the $t$-domain results in the system's averaged equations, in terms of amplitude and phase, which are given by:

$$
\begin{array}{r}
\dot{A}=\epsilon \frac{c}{2} A-\epsilon \frac{A F_{c}}{\omega_{n}}\left(\frac{\sin [\pi \Delta] \sin [\pi \Delta-2 \beta]}{(\Delta-2)}\right) \\
+\epsilon\left(0.831+A^{2}+1.657 A^{4}\right) \frac{\alpha_{2} \xi_{1}}{\omega_{n}} \sin \beta \\
+\epsilon \frac{A F_{c}}{\omega_{n}}\left(\frac{\sin [\pi \Delta] \sin [\pi \Delta+2 \beta]}{(2+\Delta)}\right)+O\left(\epsilon^{2}\right) \\
\dot{\beta}=\epsilon \sigma-\epsilon \frac{F_{c}}{\omega_{n}}\left(\frac{\cos [\pi \Delta-2 \beta] \sin [\pi \Delta]}{(\Delta-2)}\right) \\
-\epsilon \frac{F_{c}}{\omega_{n}}\left(\frac{\cos [\pi \Delta+2 \beta] \sin [\pi \Delta]}{(2+\Delta)}+\frac{\sin [2 \pi \Delta]}{\Delta}\right) \\
-\epsilon \frac{\alpha_{2} \xi_{1}}{\omega_{n}}\left(\frac{0.831}{A}+3 A+8.285 A^{3}\right) \cos \beta \\
+\epsilon \frac{\alpha_{2} A^{2} V_{d c}^{2}}{\omega_{n}}\left(2.776+7.241 A^{2}\right) \\
-\epsilon \frac{56.757 A^{2} \alpha_{1}}{\omega_{n}}+O\left(\epsilon^{2}\right)
\end{array}
$$


where $\xi_{1}=V_{d c} V_{a c}$ and $\Delta=\frac{\delta}{\omega_{n}}$. The steady-state motions occur when $\dot{A}=\dot{\beta}=0$, which corresponds to the singular points of Eqs. (26) and (27). Thus, the frequency-response can be written in its parametric form for $\beta \in[0, \pi]$ and $\Delta \in \mathbb{Q} \cap[0,1]$.

$$
\begin{aligned}
& \Omega=f_{1}(\beta, \Delta) \\
& A=f_{2}(\beta, \Delta)
\end{aligned}
$$

This analytic expression (set of two equations) makes the model suitable for MEMS and NEMS designers as a fast and efficient tool for resonant gyroscope performances optimisation.

\section{Analytical results and device specifications}

All the numerical simulations were carried out with the following set of parameters:

- Proof mass: $L_{m}=100 \mu m, e_{M}=2 \mu m, l_{c}=6 \mu m, g_{c}=2 \mu m, d_{c}=4 \mu m$ and $W_{c}=1 \mu m$.

- Resonator: $l=100 \mu m, b=2 \mu m, h=5 \mu m, g=300 \mathrm{~nm}, Q=1000$.

The estimated quality factor of the system mass-spring-damper is around $10^{5}$. The actuation voltages $V m_{a c}$ and $V m_{d c}$ are adjusted with respect to a constant $2.5 \mu m$ proof mass oscillation at resonance. The ratio between the proof mass and the resonator frequencies $(\Delta)$ as well as $V_{a c}$ and $V_{d c}$ were used for parametric studies.

\subsection{Proof mass frequency effect}

Figure 7 shows four nonlinear hardening frequency responses at a proof mass frequency ten times smaller than the resonator frequency $(\Delta=0.1)$ and for several angular rates $0-900^{\circ} / \mathrm{s}$. The $D C$ polarization of the resonator is low enough $\left(V_{d c}=1 V\right)$ to keep the global nonlinear stiffness dominated by the mechanical nonlinearities and hence, the predicted hardening behavior.

In this configuration, the Coriolis force that modulates the resonator stiffness at the proof mass frequency represents a slow dynamic with respect to the resonator dynamic ten times faster. Therefore, the frequency effect is negligible and only the resulting stress is considered which implies a positive frequency shift proportional to the external rotation rate $\theta$. Then, for $\Delta=0.1$ and for several angular rates going from $0^{\circ} / \mathrm{s}$ up to $900^{\circ} / \mathrm{s}$, the $D C$ voltage applied to the resonator is increased from $1 V$ up to $9 V$ while decreasing the $A C$ voltage from $0.2 V$ down to $30 m V$ (see Fig. 8). This increases significantly the negative nonlinear stiffness due to the electrostatic force that dominates in this case the global stiffness of the resonator and hence, the predicted softening behavior. For low actuation frequency with respect to the sensing frequency, the microgyroscope behaves as a resonant accelerometer. Indeed, the Coriolis force is seen by the resonator as a quasi-static force. Consequently, the close-form solutions of the critical amplitudes and the mixed behavior initiation amplitude established in [10] can be used here in order to improve the performances of the resonant gyroscope. Particularly, the compensation of the nonlinearities is possible when the mechanical and electrostatic critical amplitudes are equilibrated. This results on an optimal $D C$ voltage [11] for which the obtained frequency resonance peak is linear beyond the critical amplitude. 


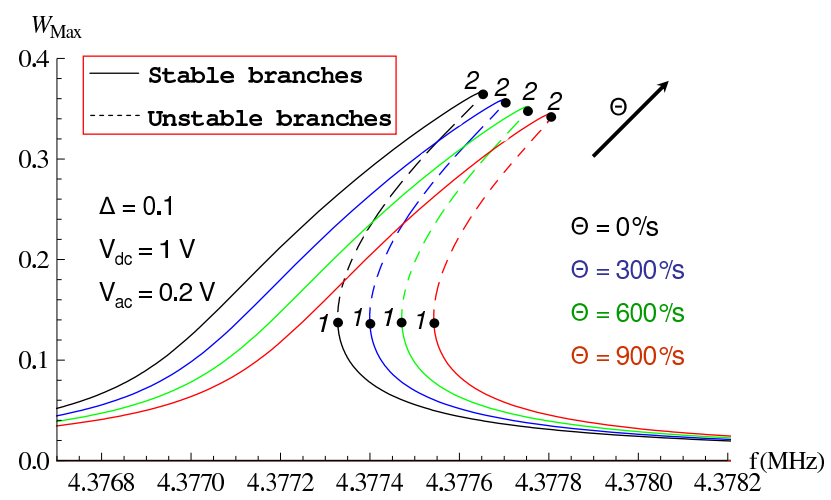

Figure 7: Predicted forced frequency responses displaying a hardening behavior for $\Delta=0.1 . W_{\max }$ is the displacement of the beam normalized by the gap $g$ at its middle point $\frac{l}{2},\{1,2\}$ are the bifurcation points. The frequency shift is due to the variation of the external angular rate $\theta$.

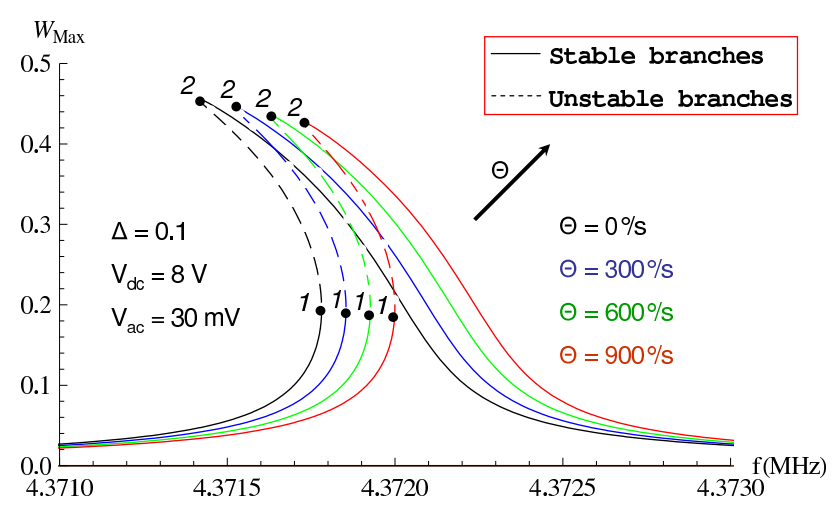

Figure 8: Predicted forced frequency responses displaying a softening behavior for $\Delta=0.1 . W_{\max }$ is the displacement of the beam normalized by the gap $g$ at its middle point $\frac{l}{2},\{1,2\}$ are the bifurcation points. The frequency shift is due to the variation of the external angular rate $\theta$. 
In a second step, the frequency of the proof mass is increased up to a quarter the resonator frequency. In the same way, for this configuration, Figures 9 and 10 show respectively several nonlinear hardening and softening forced frequency curves for several rotation rates. Thus, the compensation for the nonlinearities is possible for specific $D C$ voltage. However, the apparition of additional bifurcation points 3 and 4 is notable for external rotation rates $(\theta)$ beyond $600^{\circ} / \mathrm{s}$. The maximum is close to the bifurcation point 4 and no more situated at $\beta=\frac{\pi}{2}$. This strange behavior can be explained by an important contribution of the superharmonic resonance of order quarter the resonator primary resonance for $\theta$ beyond $600^{\circ} / \mathrm{s}$. Therefore, even out of quasiperiodicity, the averaging method is not valid except at a higher order defined by the transition curve at the corresponding superharmonic resonance. Indeed, at this level the full scale here is limited by the proof mass frequency (for a valid first order averaging).

The classical specifications of current MEMS gyroscopes include a dynamic range at best around $100^{\circ} / s$ [31] which ensures the validity of the first averaging. The curves in Fig. 11

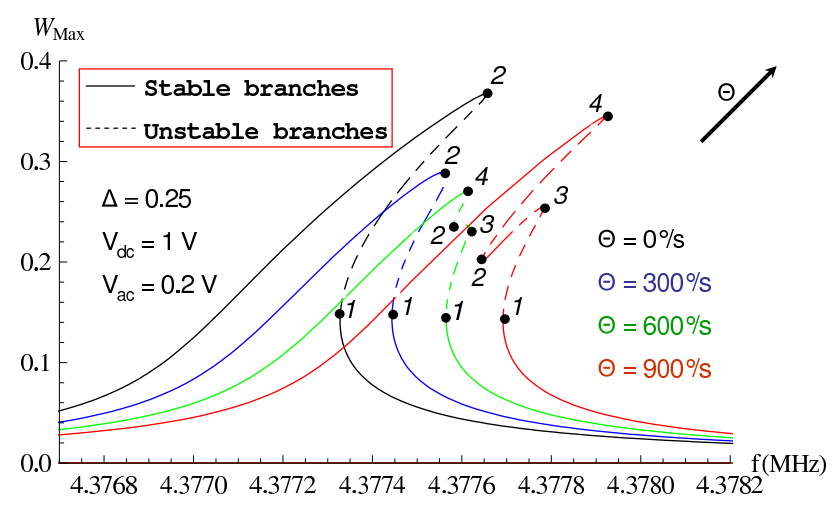

Figure 9: Predicted forced frequency responses displaying a hardening behavior for $\Delta=0.25 . W_{\max }$ is the displacement of the beam normalized by the gap $g$ at its middle point $\frac{l}{2},\{1,2,3,4\}$ are the bifurcation points. The frequency shift is due to the variation of the external angular rate $\theta$.

display the variation of the Mathieu resonator displacement at its middle point and at resonance when the phase $\beta=\frac{\pi}{2}$ for several values of angular rates $\theta$. Uncommonly, it appears that the symmetry can be broken between negative and positive Coriolis stress effect when the resonator dynamic becomes highly nonlinear for large angular rates and frequencies. This corresponds to a strong nonlinear parametric spring softening effect for which the resonator displacement averaged over the period $\frac{2 \pi}{\omega_{n}}$ is amplified (parametric perturbation) and then a high instability such as the pull-in [32] could be suddenly reached. Since the sensitivity of the resonant gyroscope could be extremely reduced for a large dynamic range, working at $\Delta<0.25$ is indispensable for high grade resonant gyroscopes.

\subsection{Resonant gyroscope scale factor}

The scale factor of the sensor, $S_{F}$ that relates the output frequency shift difference $(\delta f)$ between the two resonators to the external input rotation rate $(\theta)$ is given by:

$$
S_{F}=\frac{d f}{d \theta}\left[\frac{\Pi}{24}, \theta \in D R\right]
$$




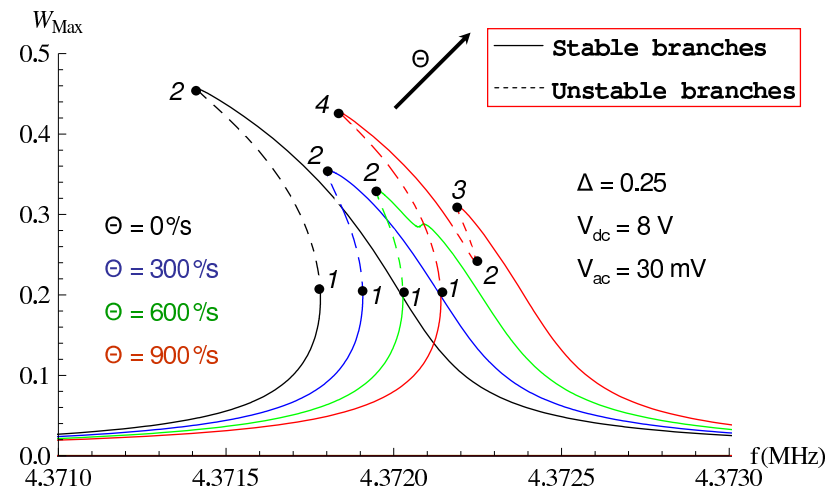

Figure 10: Predicted forced frequency responses displaying a softening behavior for $\Delta=0.25$. $W_{\text {max }}$ is the displacement of the beam normalized by the gap $g$ at its middle point $\frac{l}{2},\{1,2,3,4\}$ are the bifurcation points. The frequency shift is due to the variation of the external angular rate $\theta$.

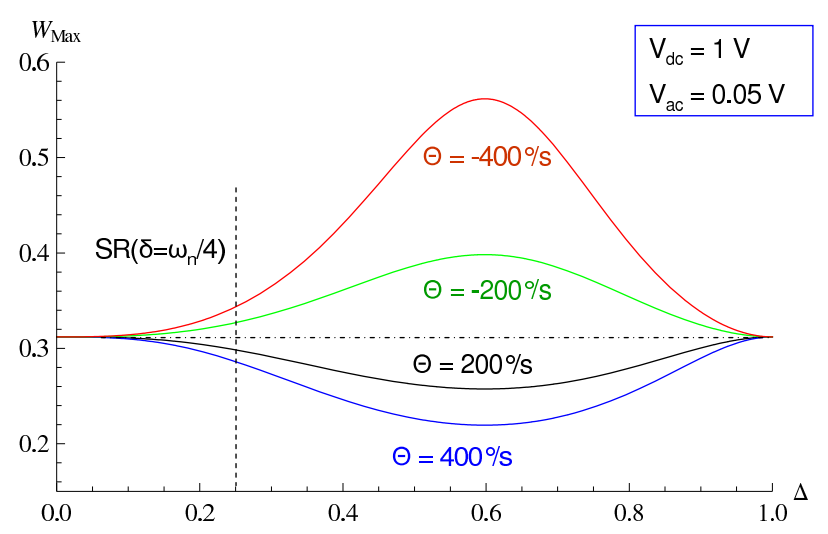

Figure 11: Variation of the Mathieu resonator displacement at its middle point and at resonance when the phase $\beta=\frac{\pi}{2}$ for several values of angular rates $\theta$. 
Note that the scale factor corresponds to the derivate of Eq. (28) at the phase $\beta=\frac{\pi}{2}$ with respect to the rotation rate $\theta$ for a dynamic range (DR) that ensures stable motions out of quasiperiodicity and negligible superharmonic resonance effects. The expression can be also written in terms of a ratio of some drive and sense parameters multiplied by a lever gain and a constant dependent on the mode shape of the resonating element. The scale factor is only dependent on material and geometrical parameters and the displacement of the gyroscope proof mass at resonance $\left(Y_{M}\right)$. Consequently and unlike classical gyroscopes, the goal of the control scheme is now simplified to the requirement of maintaining constant amplitude motion for the gyroscope proof mass and the resonator-sensing elements at their respective resonant frequencies. Figure 12 shows the variation of the resonant gyroscope scale factor with respect to the frequency ratio between the actuation and the sensing parts $(\Delta)$ for a resonator driven in the linear regime. Obviously, to use this curve, one should exclude the non-rational values of $\Delta$ (quasiperiodic regime). Remarkably, the maximum of sensitivity is located at $\Delta=0.25$ which corresponds to a superharmonic resonance in the nonlinear regime as shown in Figs. 9 and 10. Moreover the scale factor is approaching zero for three cases:

- $\Delta=0$ which is obvious since the Coriolis force is proportional to the proof mass frequency.

- $\Delta=0.5$. It corresponds to a simultaneous superharmonic and primary resonances in the nonlinear regime. In this configuration, the performances of the Mathieu resonator are drastically reduced in the linear regime and furthermore, in the nonlinear regime, the secular terms coming from the secondary resonance should be taken into account for analytical investigation in a large dynamic range.

- $\Delta=1$. It corresponds to a simultaneous parametric and primary resonance in the nonlinear regime. We arrive to the same conclusions as the case $\Delta=0.5$.

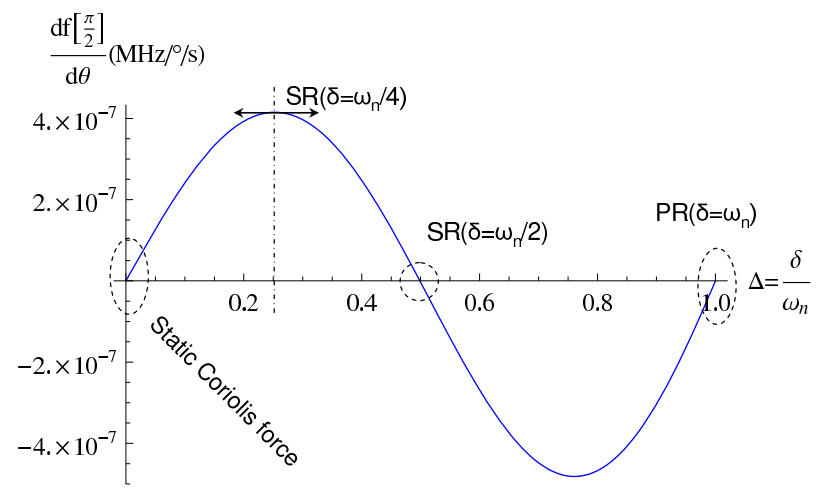

Figure 12: Scale factor variation with respect to the proof mass frequency in the resonator linear regime and inside the dynamic range of the resonant gyroscope. $S R$ and $P R$ are superharmonic and parametric resonances.

When the resonator is driven beyond its critical amplitude (in the nonlinear regime), unlike the linear case where the scale factor is constant for a given $\Delta$ and $\theta$ inside the dynamic range, the resonant gyroscope sensitivity is highly dependent on the external rotation rates for a frequency 
ratio $\delta>0.1$ as shown in Fig. 13. Curiously, the scale factor is infinitely reduced and the gyroscope becomes completely unstable if we assume a negligible secondary resonance effect which is valid up to $\Delta=0.25$ as shown in Fig. 12. Furthermore, for an admissible scale factor nonlinearity when the Mathieu resonator is driven in the nonlinear regime, the resonant gyroscope must be designed with $\Delta<0.05$.

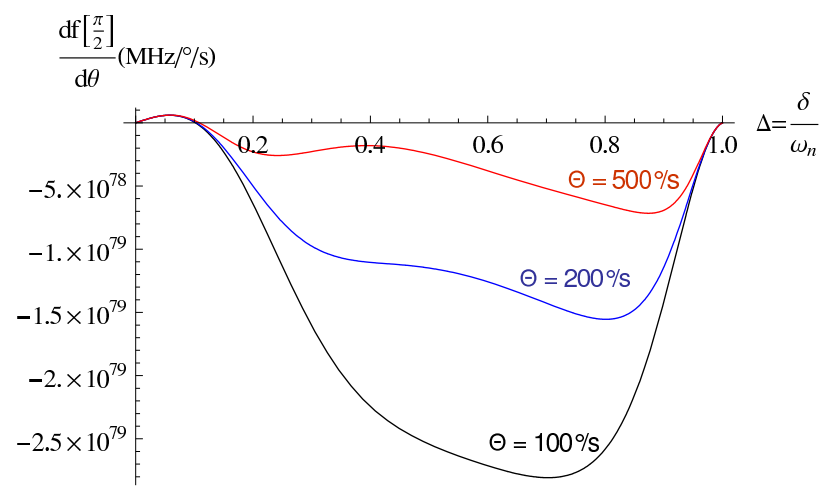

Figure 13: Scale factor variation curves with respect to the proof mass frequency for a nonlinear resonator and several rotation rates.

\section{Conclusions}

In this paper, the nonlinear dynamics of Mathieu resonator for resonant gyroscope applications has been modeled using the Galerkin discretization coupled with a perturbation technique and under few assumptions that lead to steady-state periodic motions. The relatively simple dynamic model of the nonlinear Mathieu resonator utilized here, is able to predict the measured resonator response for various parameter settings qualitatively and in many cases even quantitatively. Characteristic nonlinear dynamic steady-state behavior is very well predicted by the model. Therefore, it represents a good first step in the modeling process and a suitable starting point for understanding and predicting the dynamic behavior of resonant MEMS gyroscopes. The resulting benefits include nonlinear dynamics control, improved scale factor stability over micromechanical gyroscopes utilizing open-loop displacement sensing, large dynamic range and high resolution.

In order to provide some design rules, the variation of the gyroscope sensitivity with respect to the ratio between the proof mass and the resonator frequencies was investigated. The analytical parametric analysis showed that in the resonator linear or slightly nonlinear regimes, a frequency ratio $\Delta=0.25$ provides the greatest scale factor. However, once the resonator dynamics becomes strongly nonlinear (large oscillations beyond the critical amplitude), the sensitivity is significantly reduced if the resonator frequency is not at least an order of magnitude higher than that of the proof mass $(\Delta<0.1)$. This drastic limit could be avoided by the compensation of the nonlinearities $[10,29]$ out of quasiperiodicity for $\Delta=0.25$ which gives the maximum of sensitivity. 


\section{acknowledgments}

The authors gratefully acknowledge financial support from the CEA LETI and I@L Carnot institutes (NEMS Project) and from the ANR (M\&NEMS Project).

\section{References}

[1] N. Yazdi, F. Ayazi, K. Najafi, Micromachined inertial sensors, Proceedings of the IEEE 86 (1998) 1640-1659.

[2] J. Geen, S. Sherman, J. Chang, S. Lewis, Single-chip surface micromachined integrated gyroscope with 50 deg;/h allan deviation, Solid-State Circuits, IEEE Journal of 37 (2002) 1860-1866.

[3] W. Clark, Micromachined vibratory rate gyroscopes, Ph.D. thesis, University of California, Berkeley, 1997.

[4] N. Yazdi, F. Ayazi, K. Najafi, Micromachined inertial sensors, Proceedings of the IEEE 86 (1998) 1640-1659.

[5] S. Park, R. Horowitz, Adaptive control for the conventional mode of operation of mems gyroscopes, Journal of Microelectromechanical Systems 12 (2003) 101-108.

[6] A. Shkel, R. Horowitz, A. Seshia, S. Park, R. Howe, Dynamics and control of micromachined gyroscopes, in: American Control Conference, 1999. Proceedings of the 1999, volume 3, pp. 2119-2124.

[7] C. Acar, A. Shkel, Nonresonant micromachined gyroscopes with structural mode-decoupling, Sensors Journal, IEEE 3 (2003) 497-506.

[8] C. Acar, A. Shkel, An approach for increasing drive-mode bandwidth of mems vibratory gyroscopes, Microelectromechanical Systems, Journal of 14 (2005) 520-528.

[9] A. Seshia, R. Howe, S. Montague, An integrated microelectromechanical resonant output gyroscope, in: Micro Electro Mechanical Systems, 2002. The Fifteenth IEEE International Conference on, pp. 722-726.

[10] N. Kacem, S. Hentz, D. Pinto, B. Reig, V. Nguyen, Nonlinear dynamics of nanomechanical beam resonators: improving the performance of nems-based sensors, Nanotechnology 20 (2009) 275501.

[11] N. Kacem, J. Arcamone, F. Perez-Murano, S. Hentz, Dynamic range enhancement of nonlinear nanomechanical resonant cantilevers for highly sensitive nems gas/mass sensor applications, Journal of Micromechanics and Microengineering 20 (2010) 045023.

[12] N. Kacem, S. Baguet, S. Hentz, R. Dufour, Nonlinear phenomena in nanomechanical resonators: mechanical behaviors and physical limitations, Mécanique \& Industries 11 (2010) 521-529.

[13] N. Kacem, S. Baguet, S. Hentz, R. Dufour, Computational and quasi-analytical models for non-linear vibrations of resonant mems and nems sensors, International Journal of Non-Linear Mechanics 46 (2011) 532-542.

[14] T. Roessig, Integrated MEMS Tuning Fork Oscillators for Sensor Applications, Ph.D. thesis, University of California, Berkeley, 1998.

[15] H. Nishiyama, M. Nakamura, Capacitance of a strip capacitor, Components, Hybrids, and Manufacturing Technology, IEEE Transactions on 13 (1990) 417-423.

[16] W. I. Newman, R. H. Rand, A. L. Newman, Dynamics of a nonlinear parametrically excited partial differential equation, Chaos: An Interdisciplinary Journal of Nonlinear Science 9 (1999) 242-253.

[17] G. T. Abraham, A. Chatterjee, Approximate asymptotics for a nonlinear mathieu equation using harmonic balance based averaging, Nonlinear Dynamics 31 (2003) 347-365.

[18] A. Chatterjee, Harmonic balance based averaging: Approximate realizations of an asymptotic technique, Nonlinear Dynamics 32 (2003) 323-343.

[19] R. Rand, K. Guennoun, M. Belhaq, 2:2:1 resonance in the quasiperiodic mathieu equation, Nonlinear Dynamics 31 (2003) 367-374(8).

[20] R. Zounes, R. Rand, Global behavior of a nonlinear quasiperiodic mathieu equation, Nonlinear Dynamics 27 (2002) 87-105(19).

[21] M. Belhaq, A. Fahsi, 2:1 and 1:1 frequency-locking in fast excited van der polmathieuduffing oscillator, Nonlinear Dynamics 53 (2007) 139-152.

[22] G. Michon, L. Manin, R. G. Parker, R. Dufour, Duffing oscillator with parametric excitation: Analytical and experimental investigation on a belt-pulley system, Journal of Computational and Nonlinear Dynamics 3 (2008) 031001. 
[23] R. Dufour, A. Berlioz, Parametric instability of a beam due to axial excitations and to boundary conditions, Journal of Vibration and Acoustics 120 (1998) 461-467.

[24] Asymptotic solutions for a damped non-linear quasi-periodic mathieu equation, International Journal of NonLinear Mechanics 37 (2002) 445-460.

[25] K. Guennoun, M. Houssni, M. Belhaq, Quasi-periodic solutions and stability for a weakly damped nonlinear quasi-periodic mathieu equation, Nonlinear Dynamics 27 (2002) 211-236(26).

[26] M. Belhaq, M. Houssni, Quasi-periodic oscillations, chaos and suppression of chaos in a nonlinear oscillator driven by parametric and external excitations, Nonlinear Dynamics 18 (1999) 1-24(24).

[27] R. Rand, T. Morrison, 2:1:1 resonance in the quasi-periodic mathieu equation, Nonlinear Dynamics 40 (2005) 195-203(9).

[28] R. S. Zounes, R. H. Rand, Transition curves for the quasi-periodic mathieu equation, SIAM J. Appl. Math. 58 (1998) 1094-1115.

[29] N. Kacem, S. Hentz, Bifurcation topology tuning of a mixed behavior in nonlinear micromechanical resonators, Applied Physics Letters 95 (2009) 183104.

[30] A. H. Nayfeh, Introduction to Perturbation Techniques, Wiley, 1981.

[31] N. Kacem, Nonlinear dynamics of M\&NEMS resonant sensors: design strategies for performance enhancement, Ph.D. thesis, INSA-Lyon, CEA-LETI, Grenoble, 2010.

[32] A. H. Nayfeh, M. I. Younis, E. M. Abdel-Rahman, Dynamic pull-in phenomenon in mems resonators, Nonlinear Dynamics 48 (2007) 153-163. 